\title{
Prevalence, indication and duration of proton pump inhibitor use in patients from unselected admissions
}

\author{
Authors: Mohammed Hameed, ${ }^{A}$ Charlotte Stephens ${ }^{A}$ and Rizwan Hamer ${ }^{A}$
}

\section{Introduction}

Proton pump inhibitors (PPIs) are inhibitors of gastric acid secretion and are among the most commonly prescribed drugs worldwide. Although they are effective for treatment and prevention of peptic ulcer disease and eradication of Helicobacter pylori, there is growing evidence of adverse effects associated with PPI use. These include gastric cancer, chronic kidney disease, hyponatraemia and hypomagnesaemia, nosocomial pneumonia and recurrent Clostridium difficile infections. The National Institute for Health and Care Excellence (NICE) has published guidelines on gastrooesophageal reflux disease and dyspepsia, recommending the indications and length of treatment with PPIs. The aim of this audit was to evaluate the prevalence of PPI use, whether indication and duration was documented and explore reasons to explain the findings.

\section{Methods}

A retrospective analysis of unselected admissions of all adults lasting $\geq 24$ hours to University Hospitals Coventry and Warwickshire NHS Trust was carried out. Elective, day case, obstetrics and gynaecology admissions, and patients without a discharge summary were excluded. Pharmacists routinely carry out medicines reconciliation for all inpatients to confirm and record pre-admission medication history. Pharmacy systems and discharge summaries were used to confirm pre-admission and discharge use of PPIs, which was then compared. Electronic notes were further examined to look for indication and duration of PPI use. Finally, an online questionnaire, designed to assess the current practice of reviewing the use of PPI and to evaluate doctors' understanding of indications of PPI use, was sent to all doctors in the hospital.

\section{Results}

A total of 899 patients were discharged over five consecutive weekdays; after exclusions, 463 patients were eligible; 176 (38\%) had a PPI prescription on discharge, of whom 149 (85\%) were already on a PPI pre-admission and $27(15 \%)$ were started on a PPI in hospital. Documentation of a new prescription of PPI on discharge summaries was done in $33 \%$ of the patients; indication for PPI was documented in 33\% and duration of treatment in $22 \%$. Of the 287 (62\%) patients discharged without a PPI, 280 (98\%) were not on a PPI on admission and only seven patients had their PPI discontinued in the hospital. Median (interquartile range) earliest documented prescription to PPI use was $3(<1-6)$ years. Thirty-nine per cent of patients admitted on PPI had a documented indication; oesophagitis $(n=19 ; 31 \%)$, concurrent NSAID/steroid use $(\mathrm{n}=11 ; 18 \%)$ and gastritis $(\mathrm{n}=10 ; 16 \%)$ were the top three indications. Forty-five per cent of patients who took a PPI had an endoscopy; the median (interquartile range) was within the past $2(<1-5.5)$ years.

One hundred doctors from all grades completed the questionnaire and $35 \%$ admitted to routinely reviewing the indication for PPI for inpatients. The correct reported duration of NICE-approved indications was low and ranged from $3 \%$ to $56 \%$.

\section{Conclusion}

Over one-third of all patients admitted were prescribed a PPI, the indication and duration for which was not documented in most and the proportion in whom PPIs were stopped was very small. Numerous factors may be responsible for the low deprescribing rate of PPIs, including a lack of knowledge of NICE guidelines among doctors that may be contributing to their higher long-term use.

\section{Conflicts of interest}

None declared. 